\title{
New High-Altitude GPS Navigation Results from the Magnetospheric Multiscale Spacecraft and Simulations at Lunar Distances
}

\author{
Luke B. Winternitz, NASA Goddard Space Flight Center, \\ William A. Bamford, Emergent Space Technologies, Inc., \\ Samuel R. Price, NASA Goddard Space Flight Center
}

\section{Biographies}

\section{Luke B. Winternitz}

Luke Winternitz is a navigation systems engineer at NASA Goddard in the Components and Hardware Systems branch, where he has worked since 2001 mainly on GPS receiver research and development. He was the system architect for Magnetospheric Multiscale (MMS) GPS receivers, and currently works on the SEXTANT X-ray pulsar navigation demonstration and Restore-L navigation filter software. He received his Ph.D. (2010) in Electrical Engineering from the University of Maryland, College Park.

\section{William A. Bamford}

Bill Bamford graduated with a Ph.D. in Aerospace Engineering from the University of Texas at Austin in 2004, where he worked on a scalable platform for satellite formation state estimation and control. For the past decade he has worked with the GSFC Navigator GPS team and the GSFC Formation Flying Testbed. Additionally, he has supported a variety of high-altitude GPS programs such as MMS, Geostationary Operational Environmental Satellite (GOES) R and Orion.

\section{Samuel R. Price}

In 2009, Sam Price completed his undergraduate degree from Bradley University in Electrical Engineering. His senior capstone project focused on building a real-time software GPS receiver. Since then he has been working at Goddard Space Flight Center as the lead flight software developer for the Navigator GPS receiver, Goddard Enhanced Onboard Navigation System (GEONS), and the Station Explorer for X-ray Timing and Navigation Technology (SEXTANT) pulsar navigation demonstration.

\footnotetext{
Abstract

As reported in a companion work [13, in its first phase, NASA's 2015 highly elliptic MMS mission set a record for the highest altitude operational use of on-board GPS-based navigation, returning state estimates at 12 Earth radii. In
} 
early 2017 MMS transitioned to its second phase which doubled the apogee distance to 25 Earth radii, approaching halfway to the Moon. This paper will present results for GPS observability and navigation performance achieved in MMS Phase 2. Additionally, it will provide simulation results predicting the performance of the MMS navigation system applied to a pair of concept missions at Lunar distances. These studies will demonstrate how high-sensitivity GPS (or GNSS) receivers paired with onboard navgation software, as in MMS-Navigation system, can extend the envelope of autonomous onboard GPS navigation far from the Earth.

\section{Introduction}

Over the past decade, GPS receivers have become common, if not standard, navigation devices on Low-Earth Orbit (LEO) spacecraft. To a LEO satellite, the GPS constellation looks very similar to that of a ground user allowing early spaceborne receivers to leverage legacy GPS receiver architectures. As shown in Figure 1 this geometry breaks down quickly as the mission altitude increases. At Geosynchronous Earth Orbit (GEO) the strong mainlobe signals are only available as they spill over the limb of the Earth. To augment these signals, a new generation of receivers had been developed which could acquire and track the much weaker, but more prevalent, sidelobe signals.
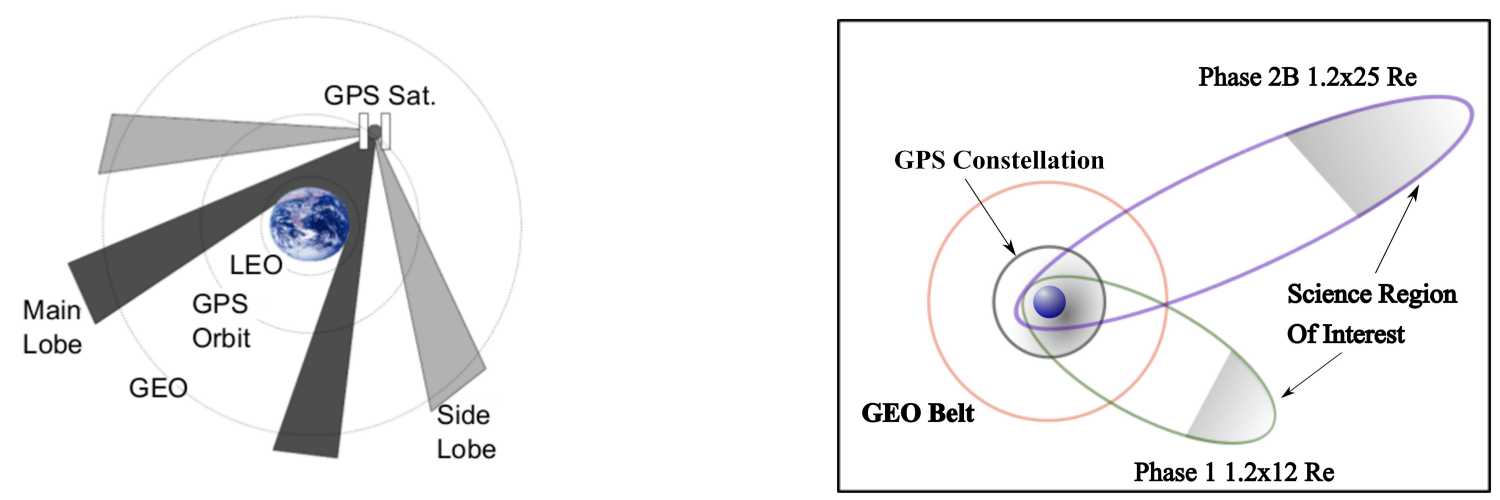

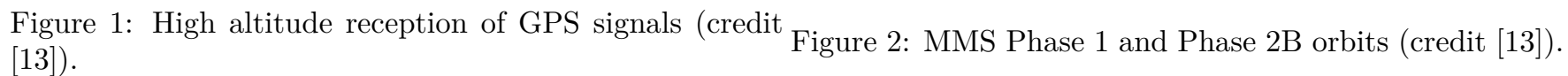

This paper is a follow-on to [13] where a detailed history of high altitude GPS receiver development was described, and navigation results from the record-setting high-altitude MMS Phase 1 were presented. The trend toward rapid development of high-altitude receivers and rapid expansion of high-altitude applications was noted there, and has continued. Notable recent progress includes the launch and successful commisioning of the GPS receiver on the Geostationary NOAA weather satellite GOES-16 (formerly GOES-R), where high-performance navigation and sidelobe tracking has also been demonstrated [12]. Additionally, further progress has been made to promote and protect signals in the the Global Positioning System (GPS) and Global Navigation Satellite System (GNSS) "Space Service Volume" [1], the volume of space between LEO and GEO.

In this paper, we briefly review the MMS mission and describe its transition (known as Phase 2A) from Phase 1 to the current orbit known as Phase 2B, with apogee at 25 Earth radii (RE), approaching halfway to the Moon. We will briefly review the MMS-navigation system consisting of the Navigator GPS receiver and integrated GEONS navigation filter and provide current flight results demonstrating its performance in Phase 2B. Finally, we will review past analyses investigating GPS navigation at Lunar distances, and present new simulation results exploring the possibility of using a potentially enhanced version of the MMS-Navigator system for two concept mission reaching Lunar distances. 


\section{MMS Navigation System Design}

Launched in 2015, MMS is a NASA heliophysics mission whose primary goal is to study the phenomenon of magnetic reconnection in the Earth's magnetosphere. It achieves this by making coordinated measurements from a large suite of instruments hosted on a precisely controlled formation of four satellites in similar highly elliptic orbits, which form a "string-of-pearls" at perigee, and a tetrahedron in the science Region of Interest (ROI) near apogee. The science mission operates in two distinct phases as shown in Figure 2 in Phase 1, the $1.2 \times 12 \mathrm{RE}$ ellipse is positioned to study the day-side magnetopause, and in Phase $2 \mathrm{~B}$, the $1.2 \times 25 \mathrm{RE}$ orbit samples the night-side neutral sheet in the magnetotail [6]. A key parameter which drives the science quality is the length of the legs of the tetrahedron. These legs could range from $7 \mathrm{~km}$ to $160 \mathrm{~km}$, and were partially constrained by the accuracy of the on-board navigation. Accordingly, a set of Orbit Determination (OD) requirements, highlighted in Table 1 were developed to ensure that the tetrahedron sizes could be maintained throughout the mission. The key requirement for Phase $2 \mathrm{~B}$ is the maintenance of the Semi-Major Axis (SMA) error to within $100 \mathrm{~m}$ when the vehicle is above $3 \mathrm{RE}$. This requirement helps to bound the temporal growth of the state propagation errors 3. Minimizing the predicitive errors decreases the frequency of formation maintainance maneuvers and maximizes the science collection intervals.

Table 1: Key MMS Phase 2B On-Board Orbit Determination Requirements (subset of table from [13]).

\begin{tabular}{l||l}
\hline Description & Requirement \\
\hline Phase 2B Semi-major Axis Estimation Req. & $100 \mathrm{~m}$ above 3 RE (99\%) \\
Orbit Position Estimation Requirement & $100 \mathrm{~km}$ Root Sum Square (RSS) (99\%) \\
PPS Distribution Accuracy & $325 \mathrm{\mu s}$ \\
Minimal Tracked Signal Level & $-175 \mathrm{dBW}$ \\
Maximum Spin Rate & 3.7 Revolutions Per Minute (RPM) \\
\hline
\end{tabular}

\section{MMS-Navigator GPS}

The Navigator GPS receiver is the product of a research and development project at Goddard Space Flight Center (GSFC) and was initiated with the goal of supporting autonomous navigation above the GPS constellation. At the time of MMS component selection, it was determined to be the only viable receiver capable of meeting the navigation requirements. Not only did the receiver have to acquire and track weak sidelobe signals, it had to do so while spinning at $3 \mathrm{RPM}$, a significant confounding factor. At the time there was also a requirement to develop and integrate a crosslink/ranging capability [13]. To handle the tracking and sensitivity requirements, while meeting spacecraft constraints, the design featured four antennas equally spaced around the perimeter. The data from each antenna was independently processed and internally switched in each channel's baseband processing to allow for handoff between antennas every five seconds. This enabled the receiver seamlessly transition between antennas as the transmitting satellite rose and set.

The MMS spaecraft are shown in Figure 3, and the GPS hardware is depicted in Figure 4. All together, MMS, has eight receivers: a primary and redundant for each of the four satellites. Each receiver is connected to one Ultra-Stable Oscillator (USO), made by Frequency Electronics, Inc., and four GPS antennas, developed in house at GSFC, with associated front-end electronics assembly, developed by Delta-Microwave, Inc.

\section{Goddard Enhanced Onboard Navigation System (GEONS)}

GEONS is an Extended Kalman Filter developed in-house at GSFC for onboard autonomous spacecraft navigation. The software had is beginnings in the 1980s with the Cosmic Background Explorer, and has matured into an invaluable tool for operational navigation and ground analysis. GEONS is currently providing onboard navigation for MMS, 


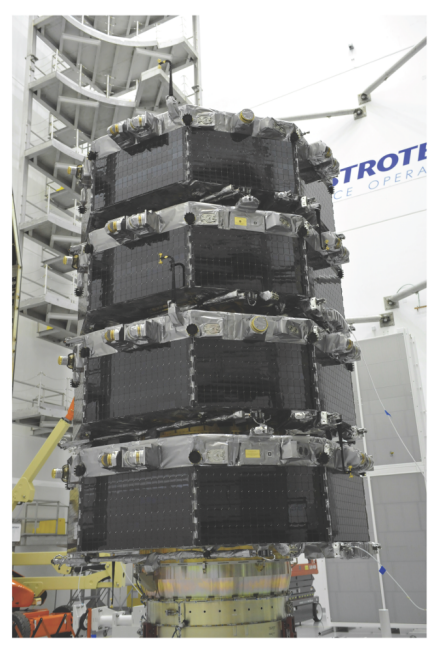

Figure 3: Stacked launch configuration of the four MMS spacecraft. The GPS antennas can be seen as black dots at each of the corners of the instrument deck (credit [13]).

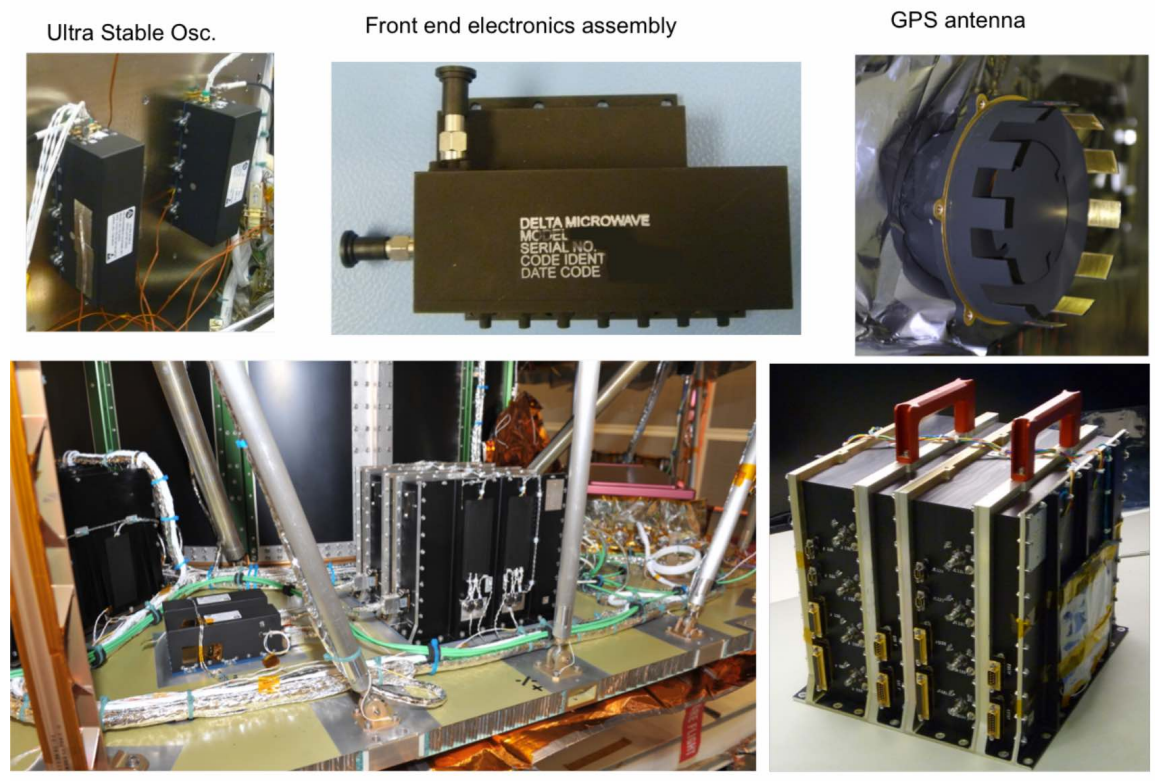

Receiver and USO on spacecraft deck

Redundant receiver electronics

Figure 4: MMS GPS hardware components (credit [13]).

the Global Precipitation Measurement mission (GPM), and Neutron-star Interior Composition Explorer (NICER) where it is instantiated twice, once to process GPS pseudorange for NICER timing, and once to process X-ray Pulsar pulse phase and frequency, as part of the Pulsar navigation demo SEXTANT. Further description of GEONS can be found in [8, 7, 13].

For MMS a nine-state filter was chosen to estimate the observatory's position and velocity, as well as the clock bias, drift, and acceleration. MMS-GEONS processes up to 12 GPS L1 C/A pseudorange measurements on a 30 second estimation cycle. The dynamics model includes a $13 \times 13$ geopotential, solar and lunar point masses, Solar Radiation Pressure (SRP) with spherical area model, and drag, and also incorporates 10-second averaged accelerations from the accelerometer during manuevers.

\section{On-Orbit Results}

After launch, the mission was inserted into an elliptic orbit which was quickly adjusted to the Phase $11.2 \times 12 \mathrm{RE}$ orbit, at that point making it the highest operational use of GPS on a spacecraft (to our knowledge). GPS navigation performance in Phase 1 was reported in [13, where predictions of performance in Phase 2B were also provided. Nearly two years after its launch, the MMS constellation raised its apogee radius to $25 \mathrm{RE}$ through a series of highly choreographed maneuvers known as Phase 2A that lasted several months. Reference [5] provides a report of navigation performance in Phase 2A. In the next section, we present GPS receiver focused results from early Phase 2B, focusing on a quiescent period without large maneuvers, during days 142-149 of 2017 encompassing about three orbits. All four primary receivers' performance has remained highly consistent with these results (no redundant receiver has needed to turn on, to date). 


\section{Receiver performance}

Figure 5a shows the number of signals tracked along with the corresponding radial distance during three orbits. Only MMS observatory 1 is shown in the plot, but the performance for the other three satellites is almost identical. As in Phase 1, the performance in Phase 2B exceeded expectations. In particular, the number of signals that the Navigator was able to track at apogee has been higher than predicted. For the orbits plotted, up to five satellites were tracked at apogee, but as many as eight have been recorded. On average about three signals are tracked near apogee, and point solutions at $25 \mathrm{RE}$ are obtained regularly. This significantly exceeds the zero, one, or two mainlobe-only signals that were predicted during pre-flight analysis, and even the two or three signals predicted in [13] after (coarsely) adjusting for Phase 1 observations. This statistic is reinforced in Figure $5 \mathrm{~b}$ which shows that, for this eight day span, at least one GPS satellite was being tracked $99 \%$ of the time and $70 \%$ of the time four or more satellites were being tracked.

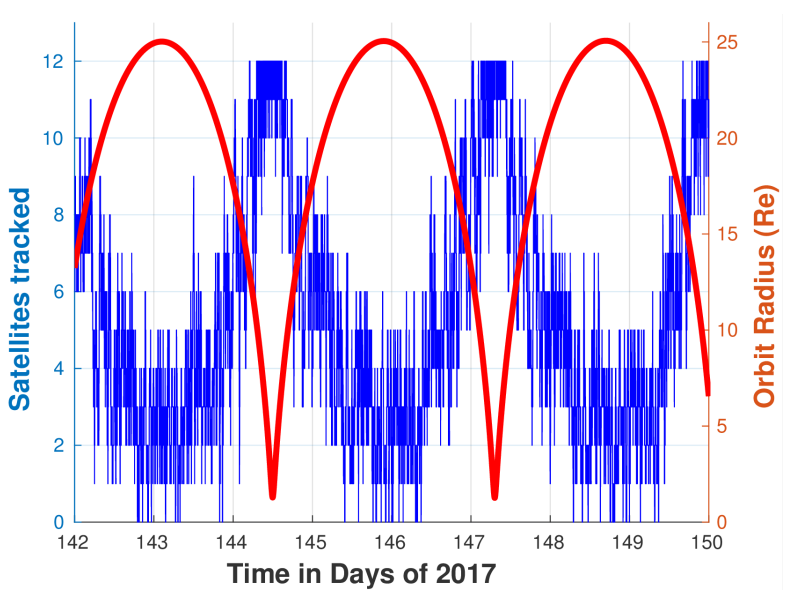

(a) Number of signals tracked and radial distance

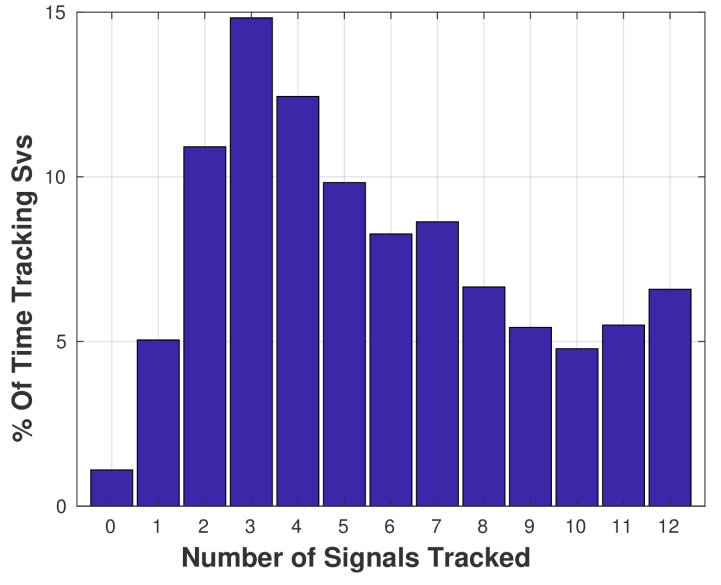

(b) Breakdown of tracked satellites

Figure 5: Number of signals tracked from days 142 through 150 of 2017

This excellent visibility above the GPS constellation is again due to Navigator's ability to acquire and track weak GPS transmitter sidelobe signals. Figure 6a and 6b (zoomed near an apogee) shows a time series of the reported $C / N_{0}$ of the GPS signals that were tracked over the same time frame as above. Aside from the peak at perigees on days 144 and 147 only sporadic main lobe signals are available, and most of the sidelobe signals never make it above $30 \mathrm{~dB}-\mathrm{Hz}$. It is these weak signals that comprise the vast majority of signals tracked at high altitude and enable the robust on-board estimation that the mission depends upon.

Figure $7 \mathrm{a}$ shows the GEONS position and velocity RSS $(1 \sigma)$ root-covariance diagonal "formal errors" and Figure 7b shows the GEONS clock bias and rate $(1 \sigma)$ formal errors from day 142 through 150 of 2017. The maximum RSS position and clock bias formal errors reach about $50 \mathrm{~m}$ just prior to returning to perigee and the maximum RSS velocity formal errors spike to a bit above $2 \mathrm{~mm} / \mathrm{s}$ near perigee entry, where the filter makes a large correction, otherwise remaining near $1 \mathrm{~mm} / \mathrm{s}$. The formal error on the clock rate is a bit higher, but remains below $6 \mathrm{~mm} / \mathrm{s}$ throughout the orbit. These errors correspond to SMA $1 \sigma$ formal error of around $15 \mathrm{~m}$, far less than the required $100 \mathrm{~m}$. As the orbit perigee in Phase 2B has increased, the range and clock errors have become highly correlated. This phenomenon can be seen in the similarity of the RSS position and the clock covariance values. This correlation will be expaneded upon further in the paper. 


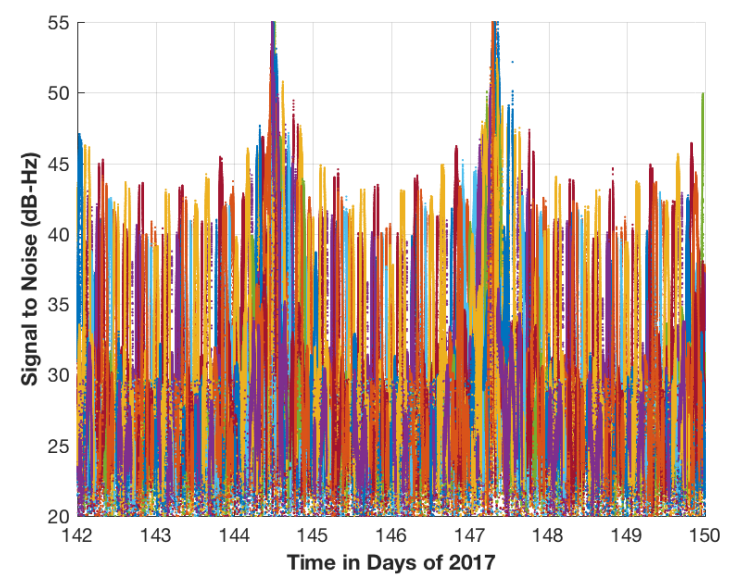

(a) Received $C / N_{0}$ for MMS observatory 1 over 8 day period starting from 2017 day 142. The majority of signals tracked at apogee are the sidelobe signals, which have $C / N_{0}$, levels between 20 and $30 \mathrm{~dB}-\mathrm{Hz}$.

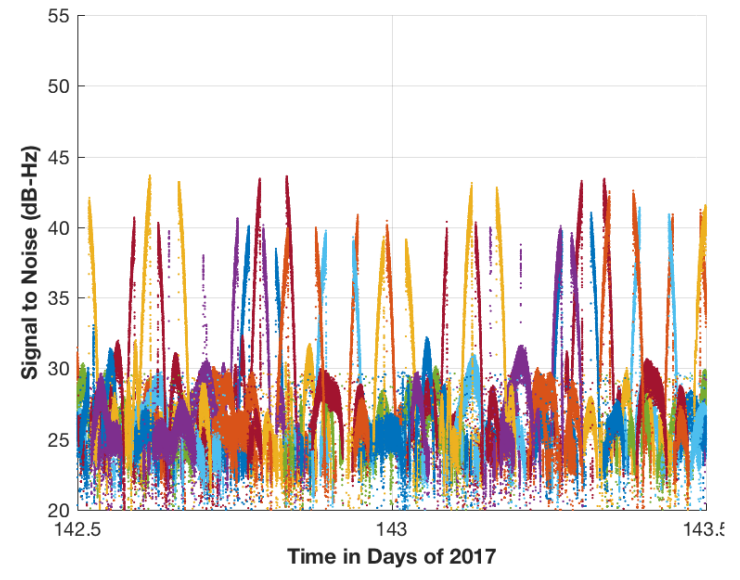

(b) Received $C / N_{0}$ as a function of time, zoomed near apogee Figure 6: Phase 2B Signal-to-Noise Ratio Plots

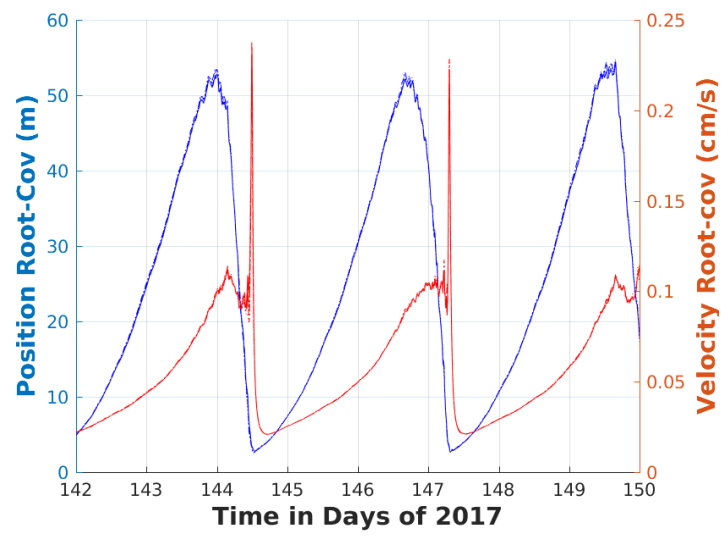

(a) RSS position and velocity

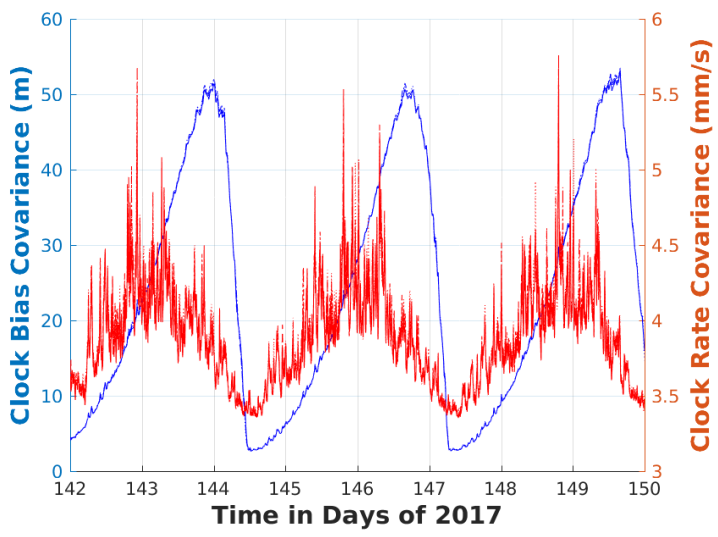

(b) Clock bias and rate. (Note the clock-rate axis unit and scaling is different than velocity plot to the left.)

Figure 7: GEONS $1 \sigma$ formal error (root-covariance) over three Phase 2B orbits. Results from each of the MMS satellites are shown, but the traces lie nearly on top of one another, due to highly consistent tracking performance.

Figures $8 \mathrm{a}$ and $8 \mathrm{~b}$ show the GEONS pseudorange residuals plotted as a function of time and true anomaly. In both plots the varying colors represent different GPS satellites tracked. At perigee, the residuals have a standard deviation of roughly $5 \mathrm{~m}$ and top out at $20 \mathrm{~m}$ at apogee.

\section{Simulations}

In this section, we present simulation results to make predictions of how well the MMS navigation system, or an enhanced version thereof, might perform in two potential future high-altitude GPS navigation applications. First, we consider one concept for an extended MMS mission that would operate after the nominal mission completes, and place at least one of the MMS observatories into an elliptical orbit with perigee similar to the MMS Phase 2B of $1.2 \mathrm{RE}$, and with apogee raised to $60+\mathrm{RE}$, at or beyond Lunar distance. We refer to this as the "MMS4" orbit. 


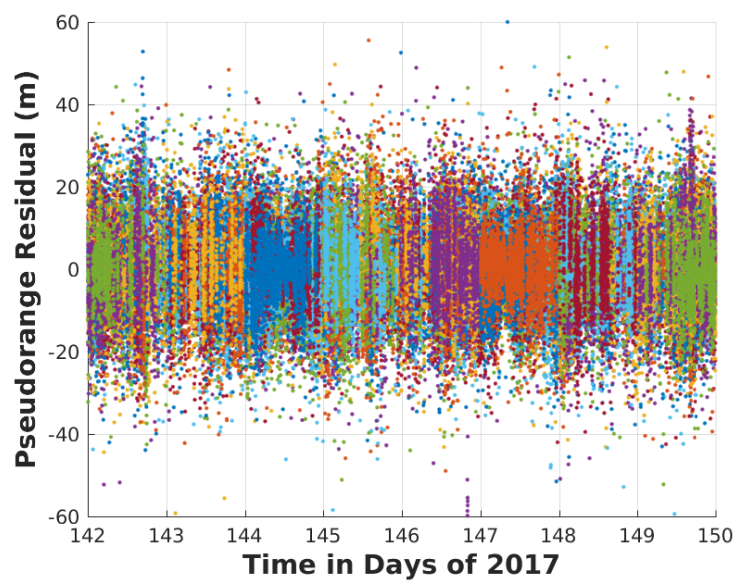

(a) vs. time

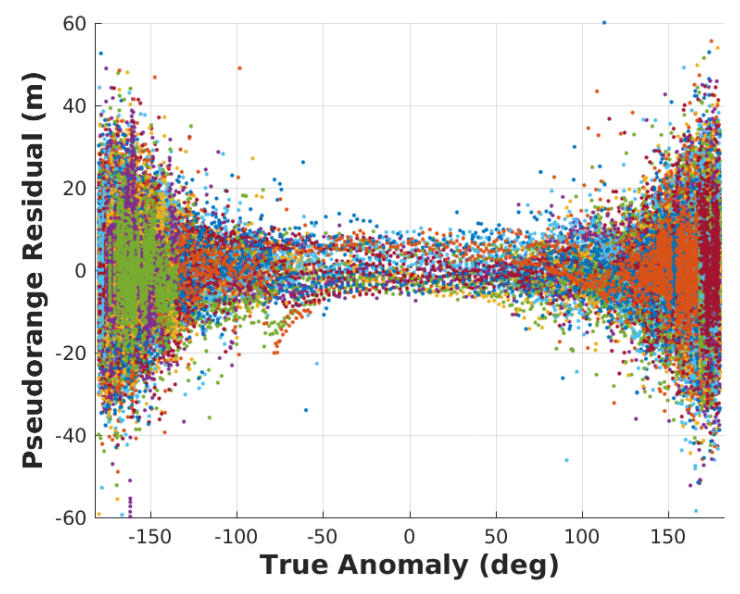

(b) vs. true anomaly $\left(180^{\circ}\right.$ apogee, $0^{\circ}$ perigee $)$

Figure 8: GEONS pseudorange residuals during day 142 to 150 of 2017

Second we consider the performance of the MMS navigation system for a hypothetical Lunar exploration mission. For this latter case, we assume an enhanced high-gain Earth pointed GPS antenna.

The two trajectories considered are shown together in Figure 9 for comparison purposes, along with the MMS Phase 2B orbit ("MMS2B") which is used for calibration of the simulation. The MMS2B and MMS4 orbits are shown in inertial coordinates, while the Lunar exploration trajectory is shown in an Earth centered frame rotating and scaled to fix the Moon's position at the mean distance from the Earth along the first axis.

\section{Selected past Lunar GPS research}

Before proceeding, we note that the concept of using GPS navigation at Lunar distances has been given significant attention by various researchers in the past. Here we briefly review selected past research.

In [2], the authors studied GPS visibility and navigation performance under various receiver sensitivity assumptions on a trans-lunar trajectory. The navigation study especially focused on fusing GPS and two-way Doppler measurements to a Lunar libration point navigation beacon satellite in a Kalman filter (in fact, they also used GEONS). The beacon helps, especially, to disambiguate the highly correlated range and clock errors which the authors note as a key challenge to navigating with GPS at lunar distances. With the beacon, the authors showed that better than 1 $\mathrm{km}$ and $5 \mathrm{~cm} / \mathrm{s}$ navigation performance was achievable even under reasonable sensitivity assumptions. These results were thought to be promising compared to some prior results.

In [14] and [9], the design and predicted performance of high-sensitivity GNSS receivers for Lunar applications. These receivers use pilot channel tracking or data wipeoff and Doppler aiding techniques to acquire and track even below 10dB-Hz, thus obtaining extremely high numbers of visible signals, but may require aiding information from the ground. The latter paper studies a multi-phase lunar mission and remarkably reports around $50 \mathrm{~m}(3 \sigma)$ accuracy for the "most challenging" descent and landing phase.

While the next-generation version of the Navigator [11, can support such very weak tracking techniques, here we focus instead on predicting the performance for a flight-proven fully-autonomous high-altitude receiver, based on a model of its current capabilities, and backed up by two-and-a-half years of flight data. 


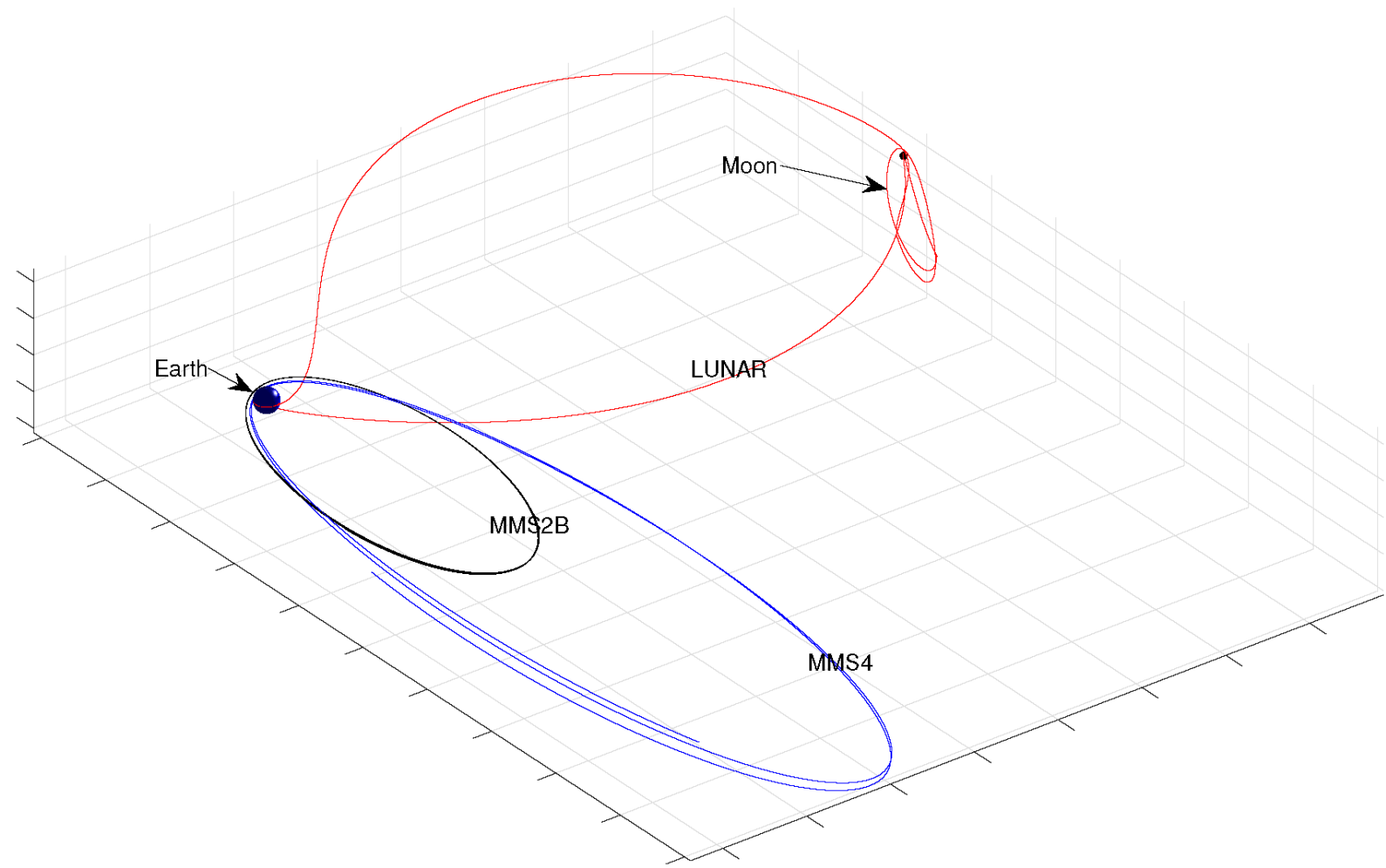

Figure 9: Trajectories considered in the simulation. The MMS2B and "MMS4" orbits are shown in inertial coordinates, while the Lunar exploration trajectory is shown in an Earth centered frame rotating and scaled to fix the Moon's position at the mean distance from the Earth along the first axis.

\section{Simulation details}

The simulations use the MMS-GEONS flight software running on the ground, and make use of an updated version of the GPS Datagen GEONS ground testing software developed to simulate GPS measurement for MMS preflight analysis. The preflight analysis is described in [10]. Rather than using the text-file-driven simulation interface that was used for MMS preflight analysis, this simulation is controlled by a more flexible GEONS ground-driver program running in MATLAB, using a recently developed GEONS MATLAB interface. We will refer to this simulation as the GEONS Ground Matlab Simulation (GGMS). The GEONS Matlab interface was developed for preflight analysis for the SEXTANT Pulsar navigation demonstration, has been further enhanced and used for Restore-L and Asteroid Redirect Robotic Mission (ARRM) analyses, and may soon be used for Wide Field Infrared Survey Telescope (WFIRST) navigation analyses. Here we setup the GGMS to run the GEONS flight software in a configuration that matches the flight configuration for the MMS/GEONS onboard software, and GPS measurement simulation that models the performance of the MMS-Navigator receiver, except when modified as described below.

\section{Calibration using MMS Phase 2B on-orbit data}

As a first step, we calibrated the GEONS ground simulation using an orbit derived from a propagated MMS2B onboard state to achieve a qualitative match to on-orbit signal visibility, signal-to-noise ratio, and covariance. We propagated forward an MMS2B on-orbit estimated state from 2017 day of year 142 for 8 days, the same period over which the on-orbit results of the earlier section were presented. We use a similar, but somewhat simplified, setup as in [10]. The truth orbit was propagated with 30x30 JGM2 Earth geopotential, point mass Moon gravity, and no 
SRP, drag, or maneuvers were modeled. A truth clock model developed for the MMS USO was used consisting of a random walk model with bias, rate, and acceleration states (with driving noise " $q$ " parameters $1.2 \mathrm{e}-22 s^{2} / s, 2.58 \mathrm{e}-$ $26 s^{2} / s^{3}$, and $1 \mathrm{e}-38 s^{2} / s^{5}$ ). (The preflight analysis considered several other deterministic and random clock effects that we have omitted here [10]). Initial errors were set randomly consistent with a covariance with Radial, In-track, Cross-track (RIC) diagonal components of $100 \mathrm{~m}, 2 \mathrm{~cm} / \mathrm{s}, 0.5 \mathrm{~s}$ clock bias, and $2 \mathrm{e}-7 \mathrm{~s} / \mathrm{s}$ clock rate, and with orbital correlations. The estimator was given the same initial covariance. The estimator uses 13x13 JGM2 gravity, and the three state clock random walk model with moderately increased $q$-parameters, to account for model uncertainty. Additional velocity process noise was added to accommodate the dynamics mismatch. The GPS measurements had $10 \mathrm{~m}(1 \sigma)$ white Gaussian noise added for signals under $40 \mathrm{~dB}-\mathrm{Hz}$ and $4 \mathrm{~m}$ noise added for those above. The link model considered path loss, a 1-dimensional model of the MMS antenna, and Earth occultation with a 1000km atmosphere mask, high enough to ignore ionosphere, and comparable to the MMS receiver atmosphere mask. To enhance simulation fidelity, a 2-dimensional transmit antenna gain model using representative Block IIR and IIR-M gain patterns from the www.gps.gov website were implimented. The transmit patterns were fixed in the satellite RIC frame with the boresight radially down. For this study, the yaw of the GPS satellites was not modeled.

In this work, we settle for a qualitative match to the on-orbit visibility and $C / N_{0}$. The current GGMS simulation does not implement a GPS transmitter yaw model, which is necessary to reproduce accurate $C / N_{0}$ passes in the sidelobes. Adding this would be a relatively simple enhancement to the simulation, and is planned for near term future work. Nonetheless, we feel the calibration obtained here is fully sufficient for making predictions of the expected performance levels that could be achieved by the MMS Navigation system in future applications.

Results of the calibration are shown in Figure 10 Figure 10a shows the number of signals tracked from the simulation, overlaid on the signals tracked on-orbit. We cannot expect to get an exact match here, but on average they agree very well. Figure $10 \mathrm{~b}$ shows an overlay of the on-orbit $C / N_{0}$ (dark blue) compared to the simulated highlighted by GPS transmitter block, showing good replication of the mainlobes and a qualitative match to the sidelobes. To obtain this calibration, we adjusted the link model receiver antenna sky noise temperature and per-block GPS transmit power (two parameters not very well characterized pre-flight) to best line up the received $C / N_{0}$ levels. The difference in the mainlobe near Earth occultation is due to the atmosphere mask of $1000 \mathrm{~km}$ in the simulation, not present in the on-orbit data (although the on-orbit filter rejects any measurement below 1000km altitude).

At the risk of some redundancy, we also present the standalone simulation results in Figure 11 for ease of comparison with results to follow. The figure on the left again has signals tracked and $C / N_{0}$, while the figure on the right has the filter $3 \sigma$ formal errors (root-covariance) and actual errors split into range and lateral components. Here we see in MMS2B the range errors dominate the lateral errors. This is due to the fact that at such high altitude as is reached by MMS2B the geometry to the GPS constellation degrades to the point where the range measurement errors and clock bias state errors become hard to distinguish. In the filter, the range and clock state errors become very highly correlated, and range knowledge is then limited by clock predictability. On-orbit formal errors (see Figure 7a) are seen to be similar to the simulated results (keeping in mind that here it shows $3 \sigma$ and previously we showed $1 \sigma$ ).

\section{MMS concept extended mission orbit}

MMS will complete its primary mission in the fall of 2017. The current plan is to extend the mission for at least two more years, during which the formation apogee will be first raised to $28 \mathrm{RE}$ [5], and eventually, in some extended mission concepts, much higher still. One concept proposes to raise apogee for at least one MMS observatory to 60+ $\mathrm{RE}$, while perigee would remain somewhere near the Phase $2 \mathrm{~B}$ orbit value of $1.2 \mathrm{RE}$. Such an orbit would have a period of approximately 20 days. Here we use the identical configuration as the calibration for MMS2B in the previous section, with a modified initial truth state to predict what level of performance might be achieved if this 

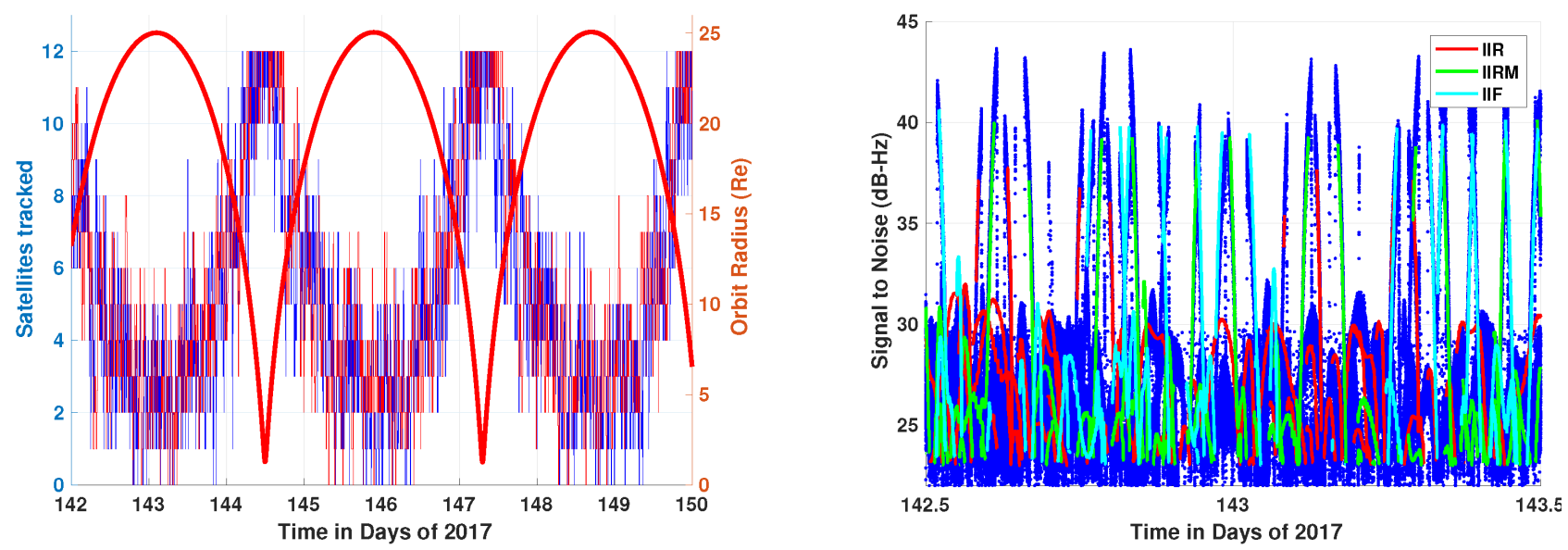

(a) Overlay of simulated number of signals tracked (red) on the (b) Overlay of simulated $C / N_{0}$ per-GPS-transmitter Block on on-orbit MMS2B data (blue), with radial distance on right axis. the on-orbit MMS2B data (blue), for a day near apogee.

Figure 10: MMS Phase 2B simulation calibration.
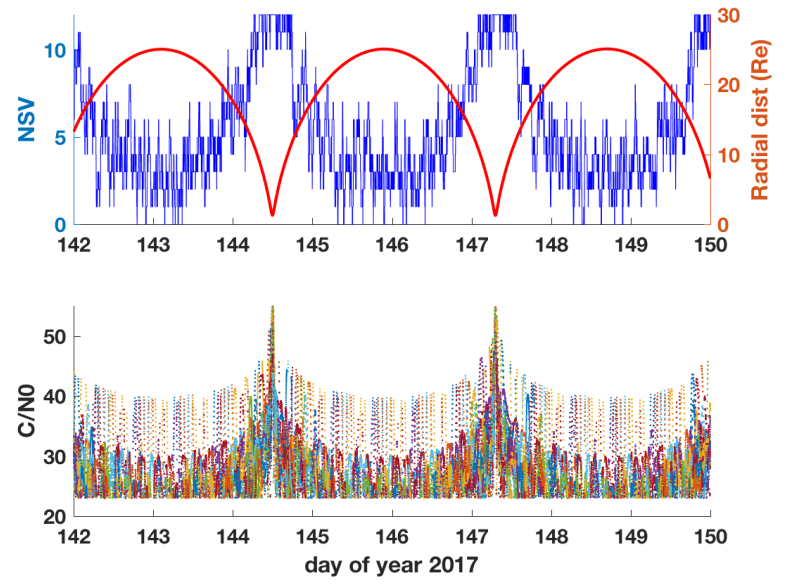

(a) Simulation number of signals tracked and $C / N_{0}$.
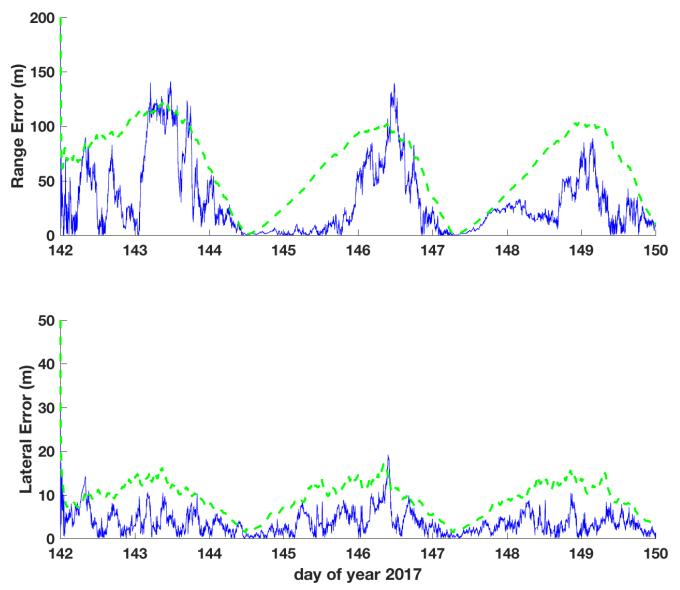

(b) Simulation on-orbit errors (solid blue line) and $3 \sigma$ rootcovariance (dashed green line) split into range/radial and RSS lateral components, showing range errors dominate due to clock uncertainty, not shown, but which are nearly the same.

Figure 11: Simulation of MMS Phase 2B

concept were executed.

The results are shown in Figures 12a and 12b As in MMS2B, but to a increased extent, the clock bias and range error become very highly correlated. Clock and range errors are at the 1-2 kilometer level, while the lateral component of position are an order of magnitude better. For future missions, the range/clock issue could be resolved by using an improved (namely, an atomic) clock, or by providing the filter with a clock-independent measurement, such as two-way range and/or Doppler as in [2].

\section{Concept Lunar exploration trajectory}

NASA's human exploration will return astronauts to the Moon with for the first time since Apollo with its Orion spacecraft in the near future starting with Exploration Missions 2 and/or 3. Concepts for Lunar or Lunar libration 

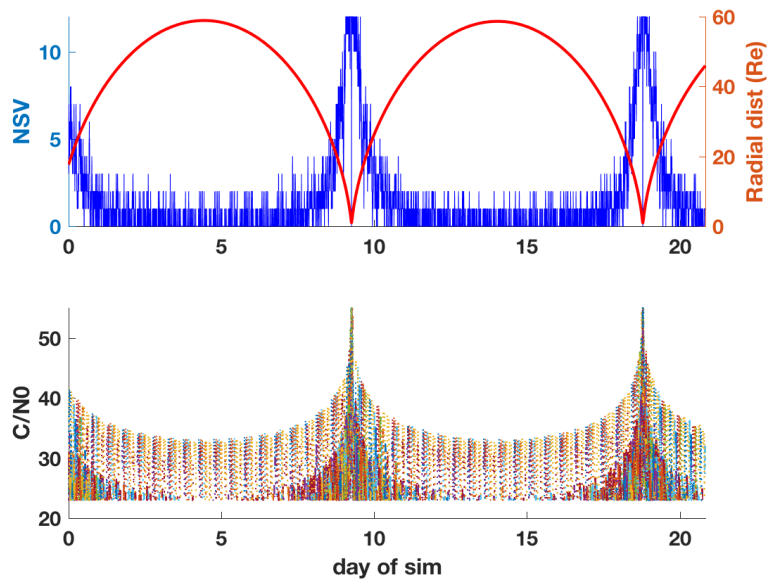

(a) Predicted visibility (top) and $C / N_{0}$ (bottom) for enhanced MMS mission "Phase 4" orbit reaching apogee distances at or beyond Lunar distance. The red curve on the right axis of the top plot shows the radial distance from Earth.
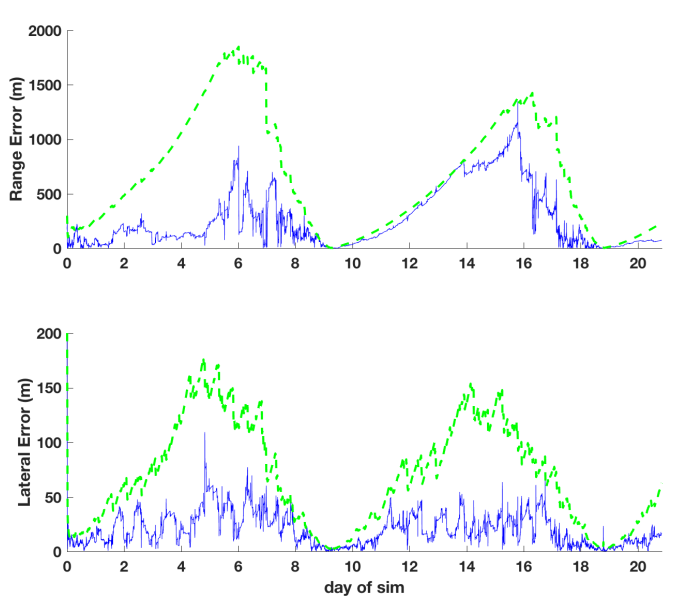

(b) Predicted performance: position errors (solid blue line) and $3 \sigma$ root-covariance (dashed green line) separated into radial and lateral directions.

Figure 12: MMS "Phase 4" Simulation Results

point orbiting space stations may also be on the horizon.

In this section we simulate the performance of the MMS-Navigation system, paired with a high-gain antenna, for a trajectory that may be representative of a future Lunar exploration mission. The trajectory starts in LEO, then transfers impulsively to a trans-lunar trajectory, enters a Near Rectilinear Halo Orbit (NRHO), which are generally of interest for human exploration missions [4, for a couple of orbits then transfers back to the Earth. The receiver model is identical to that of the MMS-Navigator in the prior two sections except for the receiver antenna. Here we consider switching from a $4 \mathrm{dBi}$ antenna to a $14 \mathrm{dBi}$ high-gain at $12 \mathrm{RE}$ on the outbound trajectory and switching back on the return trajectory at the same distance. We effectively assume the antenna remains pointed back at the Earth over the duration of the mission by using an isotropic receiver gain pattern in the simulation. We use the same dynamics assumptions as in the previous section, and same filter configuration, except we provided the true delta- $\mathrm{V}$ impulses to GEONS, with process noise increased near maneuver times. Apart from maneuvers, we significantly increased the velocity process noise baseline, and further increased it during near-Moon passes to handle a mismatch in the modelling of the (non-spherical) Moon gravity truth model and the filter model (point mass), which initially caused problems during close passes to the Moon.

Signal tracking results are shown in Figure 13a As expected, thanks to the high-gain antenna, the visibility is similar to that of MMS2B, with the additional path loss made up for by the additional antenna gain. Sidelobes are tracked at the Moon and it is common for more than four signals to be tracked simultaneously. Navigation performance is shown in Figure 13b. Here we obtain better than $1.5 \mathrm{~km}$ range/clock $3 \sigma$ formal (and actual) errors and $100 \mathrm{~m}$ level $3 \sigma$ RSS lateral errors. For some applications this may be fully adequate as is. In other cases, performance could be greatly improved through use of an atomic clock, or addition of a periodic clock-independent measurement source.

\section{Conclusion}

MMS results from Phase 1, presented in [13, and especially from the Phase 2B data presented here, coupled with results from other missions, such as GOES-16, have shown that GPS navigation at high-altitude can provide excellent performance, simplify operations, and provide cost benefits to missions. We believe it is also safe to say that we have 

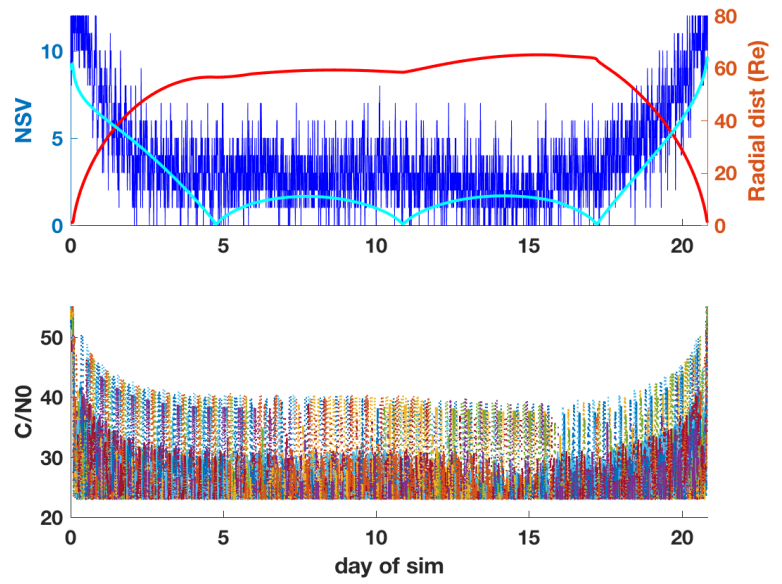

(a) Predicted visibility (top) and $C / N_{0}$ (bottom) for the Lunar exploration trajectory. The red curve on the right axis of the top figure is the radial distance from Earth reaching from 0 to $60+\mathrm{RE}$, the blue curve on the same axis is the radial distance from the Moon, also in RE.
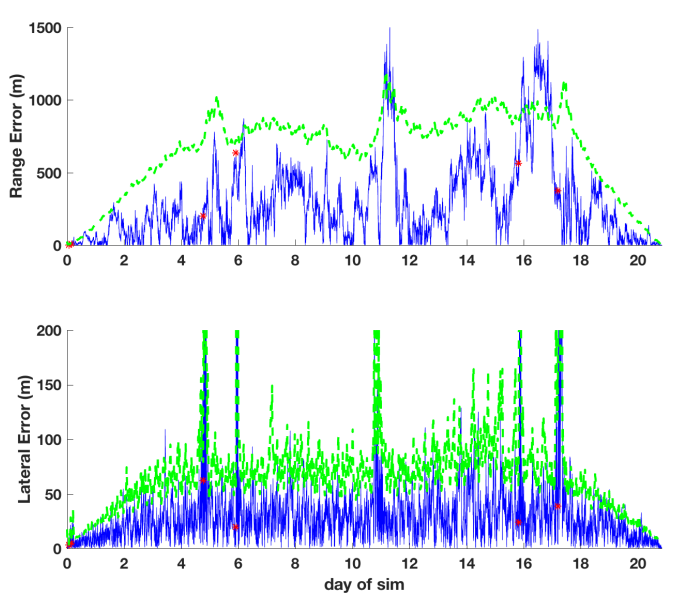

(b) Predicted performance: position errors (solid blue line) and $3 \sigma$ root-covariance (dashed green line) separated into radial and lateral directions for the Lunar exploration trajectory. Maneuvers are shown as red asterisks.

Figure 13: Simulation of Lunar Exploration Mission

not yet reached the altitude limit where GPS navigation remains practical.

In the near future, new GNSS systems and signals will be coming online with receivers able to take full advantage of the enhanced coverage and signal performance they will offer. This future technology is certainly bound to help push the envelope of performance in challenging applications such as very high-altitude GPS navigation. In the simple simulations presented here, we have attempted to show that strong autonomous, on-board, GPS navigation performance levels at Lunar distances is achievable now using currently available GPS signals, and flight proven receiver technology. In particular, we believe, the MMS-Navigation system, with a high-gain antenna, can provide strong navigation performance at Lunar distances.

\section{Acknowledgment}

The authors wish to acknowledge the work of the MMS Navigator and Flight Dynamics teams and other navigators at GSFC who have contributed years of hard work to help make this program a success. We would also like to thank Russell Carpenter, Anne Long, and Ben Ashman for helpful discussions and feedback on the paper, and Chris D'Souza for providing helpful information on Lunar navigation topics.

\section{References}

[1] Frank H. Bauer, Joel J. K. Parker, Bryan Welch, and Werner Enderle. Developing a robust, interoperable GNSS space service volume for the global space user community. In Proceedings of the Institute of Navigation International Technical Meeting, January 2017.

[2] J. Russell Carpenter, David C. Folta, Michael C. Moreau, and David A. Quinn. Libration point navigation concepts supporting the vision for space exploration. In AIAA/AAS Astrodynamics Specialist Conference. AIAA, 2004. 
[3] J. Russell Carpenter and Emil R. Schiesser. Semimajor axis knowledge and GPS orbit determination. NAVIGATION: Journal of The Institute of Navigation, 48(1):57-68, Spring 2001. Also appears as AAS Paper 99-190.

[4] Diane Davis, Sagar Bhatt, Kathleen Howell, Jiann-Woei Jang, Ryan Whitley, Fred Clark, Davide Guzzetti, Emily Zimovan, and Gregg Barton. Orbit maintenance and navigation of human spacecraft at cislunar near rectilinear halo orbits. In Proceedings of the AIAA SPACE Conference 2016, September 2017.

[5] Mitra Farahmand, Anne Long, Jacob Hollister, Julie Rose, and Dominic Godine. Magnetospheric multiscale mission navigation performance during apogee-raising and beyond. In Proceedings of the AAS/AIAA Astrodynamics Specialist Conference, August 2017.

[6] Cheryl Gramling. Overview of the magnetospheric multiscale mission. In Proceedings of the AAS Astrodynamics Specialist Conference, Pittsburgh, PA, August, 2009.

[7] Cheryl Gramling, J. Russell Carpenter, Anne Long, David Kelbel, and Taesul Lee. Autonomous relative navigation for formation-flying satellites using GPS. In 15th International Symposium on Space Flight Dynamics, Toulouse, France, 2000. CNES. Paper MS00/18.

[8] Anne Long, David Kelbel, Taesul Lee, James Garrison, and J. Russell Carpenter. Autonomous navigation improvements for high-earth orbiters using GPS. In 15th International Symposium on Space Flight Dynamics, Toulouse, France, 2000. CNES. Paper MS00/13.

[9] Luciano Musumecia, Fabio Dovis, Joao S. Silva, PedroF. da Silva, and Hugo D.Lopes. Design of a high sensitivity GNSS receiver for lunar missions. Advances in Space Research, 57:2285-2313, June 2016.

[10] Corwin Olson, Cinnamon Wright, and Anne Long. Expected navigation flight performance for the magnetospheric multiscale mission. In Proceedings of the 22nd AAS/AIAA Conference, Charleston, SC, January, 2012.

[11] David Petrick, Nat Gill, Munther Hasouneh, Robert Stone, Luke Winternitz, Luke Thomas, Milton Davis, Pietro Sparacino, and Thomas Flatley. Adapting the SpaceCube v2.0 data processing system for mission-unique application requirements. In Proceedings of the NASA/ESA Conference on Adaptive Hardware and Systems, June 2015.

[12] Stephen Winkler, Graeme Ramsey, Charles Frey, Jim Chapel, Donald Chu, Douglas Feesland, Alexander Krimchansky, and Marco Concha. GPS receiver on-orbit performance for the GOES-R spacecraft. In Proceedings of the 10th International ESA Conference on GNC Systems, May 2017.

[13] Luke B. Winternitz, William A. Bamford, Samuel R. Price, J. Russel Carpenter, Anne C. Long, and Mitra Farahmand. Global positioning system navigation above 76,000 km for NASA's magnetospheric multiscale mission. NAVIGATION, Journal of The Institute of Navigation, 64(2):289-300, August 2017.

[14] Norbert Witternigg, Gunther Obertaxer, Michael Schonhuber, Giovanni B. Palmerini, Filippo Rodriguez, Luigi Capponi, Francis Soualle, and Jean-Jacques Floch. Weak GNSS signal navigation for lunar exploration missions. In Proceedings of the 28th International Technical Meeting of The Satellite Division of the Institute of Navigation, pages 3928-3944, September 2015. 